\title{
Varones víctimas de violencia doméstica: un estudio exploratorio acerca de su percepción y aceptación*
}

\section{Men victims of domestic violence: an exploratory study about perception and acceptance}

\author{
Patricia Trujano** \\ Aimé Edith Martínez \\ Samanta Inés Camacho \\ Universidad Nacional Autónoma de \\ México \\ Recibido: 19 de mayo de 2009 \\ Revisado: 2 de julio de 2009 \\ Aceptado: 30 de agosto de 2009

\section{Resumen} \\ Un hecho novedoso en el fenómeno de la violencia \\ doméstica es el incremento de denuncias de varo- \\ nes en contra de sus mujeres, pero existe muy poca \\ investigación al respecto. Nuestro objetivo fue iden- \\ tificar en cien varones heterosexuales, qué actitudes \\ y comportamientos percibían como violentos en su \\ pareja, así como la frecuencia y modalidades con que \\ se presentaban. Cincuenta de ellos mantenían una re- \\ lación de noviazgo y cincuenta eran casados. Respon- \\ dieron al inventario VIDOFyP en la ciudad de México. \\ La violencia estuvo presente en ambas muestras con \\ niveles bajos, pero hubo una mayor incidencia y una \\ mayor percepción en los casados. Las modalidades \\ con mayor frecuencia y mejor percibidas por ambos \\ grupos incluyeron la psicológica, social y sexual. Per- \\ cepción y frecuencia correlacionaron positivamente, \\ sugiriendo que la sola discriminación de actitudes y \\ comportamientos violentos no es suficiente para evi- \\ tarlos. \\ Palabras clave: violencia de género, violencia do- \\ méstica, varones maltratados, noviazgo, matrimonio. \footnotetext{
lleva a cabo en la Facultad de Estudios Superiores Iztacala, Universidad Nacional Autónoma de México.

** Correspondencia: Patricia Trujano. Facultad de Estudios Superiores Iztacala. Universidad Nacional Autónoma de México. Dirección postal: Carrera de Psicología. Ave. De los Barrios No. 1, Los Reyes Iztacala, Tlalnepantla, CP 54090, Estado de México, México. Correo electrónico: trujano@ servidor.unam.mx
} \\ Este trabajo forma parte de una amplia investigación titulada: Violencia: género, masculinidad y violencia doméstica hacia el varón, que se
}




\section{Abstract}

A novel fact in the phenomenon of the domestic violence is the men who begin to denounce they are victims of their women, but at the moment exists very little investigation. Our objective was to identify in 100 heterosexual men what attitudes and behaviors perceived like violent in their pair as well as the frequency and modalities whereupon appeared: 50 of them with a relation of engagement and 50 married. VIDOFyP inventory was applied in Mexico's City. Low violence levels were found on both samples, but there was a greater incidence and a greater perception in married men. The modalities with a greater incidence and better perceived by both groups were psychological, social and sexual. A positive correlation between perception and frequency was observed, suggesting that the discrimination of attitudes and violent behaviors is not enough so that they are able to be avoided.

Key words: gender violence, domestic violence, mistreated men, engagement, marriage.

"[...] la sociedad no tiene derecho a discriminar a una víctima de violencia doméstica debido a su género[...]"

www.batteredmen.com

\section{Introducción}

La percepción social generalizada acerca de la violencia doméstica es que ésta tiene siempre como víctimas a las mujeres y como agresores a los hombres, lo que ha favorecido el surgimiento de medidas y declaraciones que, aunque tienen la intención de acabar con la violencia doméstica (también aluden a ella como violencia de género), mantienen un sesgo importante que otorga al varón invariablemente el papel de verdugo (Pérez, 2005).

Incluso, agencias internacionales como la Organización Mundial de la Salud (OMS) o leyes de suma trascendencia por la protección que ofrecen a las víctimas (como la Ley Integral contra la Violencia de Género en España que viera la luz en diciembre del 2004, o la Ley General de Acceso de las Mujeres a una Vida Libre de Violencia, promulgada en México en febrero del 2007) mantienen esta postura, lo que parece una actitud sexista, discriminatoria y sobre todo, lejana de la tan buscada equidad de género.

Nosotros pensamos que las leyes de protección a las víctimas deberían ser lo suficientemente incluyentes para todos, especialmente si consideramos temas pendientes como las nuevas relaciones que se están gestando de manera cada vez más abierta en las sociedades actuales. Como señala Vílchez (2007), hoy por hoy, hablar de la violencia de la mujer contra el varón no es políticamente correcto y, por el contrario, puede restar votos y simpatías de muchas mujeres, incluidos los grupos feministas, además de acarrear acusaciones por machismo o misoginia, y subraya que las denuncias suelen registrar sólo la violencia doméstica contra la mujer, mostrando la mitad del problema. Pues es verdad que, desde los formatos de registro, los protocolos de atención y la infraestructura generada está orientada a ellas, como las comisarías y agencias especializadas, las casas refugio, las líneas de apoyo telefónico, etcétera.

Veamos algunas cifras, recordando que es difícil la precisión, y que, como sucede con las mujeres, pueden ser sólo la punta del iceberg. Algunos autores en 1997 encontraron que el 1\% de las esposas maltrataba a sus maridos (Olamendi, 1997); para 1998 otros observaron un 2\% (Corsi, 1997; 1998); pero para el año 2005, se hablaba ya de al menos un $10 \%$ a $15 \%$ de varones golpeados. $\mathrm{Fi}$ nalmente, se calcula un $23 \%$ aproximadamente en que los combates son mutuos; al respecto, por razones obvias, existe menos investigación.

En México tenemos una dificultad adicional, y es que cada fuente tiene sus propias estadísticas, que suelen no coincidir del todo; éstas pueden consultarse en las páginas web de las instituciones oficiales: el Centro de Atención a la Violencia Intrafamiliar (CAVI) informó que en el primer 
semestre de 1997, una de cada 10 víctimas de violencia doméstica era varón; pero para el 2006 aumentó al $14.1 \%$ en la capital de la República, según el mismo CAVI y el DIF (Desarrollo Integral para la Familia).

En Guanajuato, datos recientes revelan que el 10\% de las demandas corresponden a víctimas hombres. Durante el año 2001 se registró un 6\% de varones víctimas de sus parejas, y de enero a agosto del 2002, hubo 23 denuncias por parte de ellos en el Estado de Aguascalientes. También en el D.F., de enero a septiembre del 2002, fueron atendidos por violencia doméstica de sus cónyuges 234 varones, según cifras de las Unidades de Atención y Prevención de la Violencia Familiar (UAPVIF).

Algunos estudios revelaron que de cada siete hombres denunciados en el 2003 como agresores, tres eran en realidad las víctimas (Del Ángel, 2003). En el mismo 2003, el Consejo General del Poder Judicial en México informó de siete varones asesinados por sus esposas. En el 2004, el Instituto Nacional de las Mujeres confirmó que 73 varones fueron atendidos por malos tratos sólo en el D.F. y en el 2005, en Veracruz se presentaron 56 denuncias de varones por malos tratos físicos y psicológicos. En el mismo año, las personas atendidas por violencia familiar en las unidades de la Secretaría de Salud del Distrito Federal (SSDF) manifestaron que el $21.8 \%$ de las víctimas fueron varones. En el 2007, el Procurador General de Justicia en el Estado de México aseguró que en esta entidad, en los últimos años por cada asesinato de mujeres ocurren tres homicidios dolosos contra hombres, casi todos producto de problemas pasionales.

Con respecto a otros países, en Bolivia se registraron trece mil denuncias por maltrato doméstico durante el año 2004; más de 1.000 eran de varones. En el 2007, la Brigada de Protección a la Familia habla de seis víctimas masculinas cada día.

En Perú, de acuerdo con el Programa Nacional contra la Violencia Familiar y Sexual, las estadísticas indican una creciente proporción y vulnerabilidad de varones víctimas de la violencia conforme aumenta su edad. Según datos del 2002, la principal persona agresora en el grupo compren- dido entre los 18 y 59 años lo constituye la pareja conviviente o esposa.

En España, en el año 2000 fueron asesinados 44 hombres por sus esposas; 16 más en el 2002. En el 2005 la cifra fue de quince. Pero si contamos a todos los varones muertos en el ámbito familiar por cónyuge, excónyuge, novia, compañera sentimental, hijo/a, padre/madre, o pariente, tenemos que suman 56 , representando el $48.2 \%$ de la cifra total, según el Ministerio del Interior, Anuario 2005. En el mismo año, en Andalucía se registraron 2.600 casos de varones maltratados por sus mujeres y la Fiscalía de Madrid, en el 2006, informó que se perpetraron sólo en esa Comunidad 2.589 delitos de violencia cometidos por mujeres en contra de sus parejas (Montero, 2007). En el resto de Europa, países como Francia, Alemania y Noruega parecen tener las cifras más altas de violencia doméstica en contra de los varones.

En Estados Unidos existe más investigación al respecto: de acuerdo con cifras de su Departamento de Justicia, cada año más de ochocientos mil varones son víctimas del maltrato de sus mujeres. Datos de Washington apuntan que cada 37 segundos un hombre es herido por su compañera con pistolas, cuchillos u otros objetos, además de ser frecuentes las lesiones por patadas, rasguños, mordiscos, y jalones de cabello. Autores como Eloy Rodríguez (citado en Bernal, 2001) aseguran que en la actualidad, en Estados Unidos la cifra de muertes de hombres por violencia doméstica es mayor que la de las mujeres. Ciertamente, pensamos que los varones deben enfrentar aun mayores dificultades para obtener credibilidad, de acuerdo con los testimonios que conocemos.

Si nos detenemos a analizar el patrón de violencia ejercido por las mujeres, las observaciones de los psicólogos apuntan a que es similar cuando ellas son las víctimas, lo que puede llegar al asesinato. Es decir, ellos también sufren violencia física, psicológica, sexual, económica, social y objetal: algunas esposas maltratadoras se burlan en privado o en público del marido, lo intimidan y humillan; lo aíslan de familiares y amigos; le retienen el dinero; lo amenazan con suicidarse o dañar a sus hijos; le impiden trabajar o estudiar; lo chantajean 
con gritar pidiendo ayuda a los vecinos, seguras de que les creerán a ellas; lo agreden físicamente de propia mano o recurren a terceros (a través de familiares, amigos o amantes) a quienes convencen de que el marido merece ser castigado (Del Ángel, 2003; Monserrat, 2006).

Por su parte, Hoff (1999) agrega otros indicadores, como intentar vigilar y acaparar todo su tiempo, acusarlo constantemente de infidelidad, enojarse fácilmente, destruirle sus propiedades o cosas con valor sentimental, pegarle, darle bofetadas, patearlo, morderlo, arrancarle cabello, rasguñarlo, amenazar con herirlo a él o a sus hijos e incluso forzarlo a tener sexo contra su voluntad. Esto lo sostienen diversos autores como Olszewski (1999), Padres por la lgualdad Parental (2000) y Pérez (2006). Llerena (2004) asegura que de cada cien varones, cuarenta son agredidos por sus mujeres, y Menacho (2004) subraya la tendencia a violentar psicológicamente a los hombres cuestionando su eficacia sexual.

Fiebert (2004), al examinar 244 estudios sobre la violencia conyugal en todo el mundo, concluyó que las mujeres son significativamente más propensas que los hombres a expresar violencia, que el $29 \%$ de ellas reconocen haber agredido a sus parejas en los últimos cinco años, y que tienen tres veces más probabilidades de usar un arma que un hombre en el curso de un conflicto marital. Este mismo autor, profesor de Psicología de la Universidad de California, muestra también resultados producidos a través de 147 investigaciones especializadas, 119 estudios empíricos y 28 análisis efectuados, lo que finalmente lo lleva al examen de más de 106.000 casos, lo que debilita el argumento de que sólo existen especulaciones y testimonios cuestionables sobre el tema. Algunos autores hacen ya intentos por reunir y formalizar la información actual (Trujano, 2008).

Paralelamente, en lo que se refiere a la dinámica de estas relaciones, llaman la atención los estudios que afirman haber observado en las mujeres un cuadro de ejercicio de la violencia similar al de los hombres, con una elevación significativa con el paso del tiempo en la escalada de episo- dios violentos, un carácter cíclico (que suele iniciar con una etapa de acumulación de tensión, seguida de la crisis o descarga aguda de violencia) $y$, por último, una fase llamada luna de miel o reconciliatoria, que paradójicamente puede ser el anuncio de que se reinicia dicho ciclo (Walker, 1984) y en los hombres que son las víctimas, aparecen un conjunto de reacciones al maltrato como el que pueden mostrar ellas (síntomas derivados reconocidos como "Síndrome de la Mujer Maltratada"), lo que incluye una gran dificultad para buscar ayuda y abandonar la relación (Hoff \& Easterbrooks, 1999).

Algunas investigaciones en Estados Unidos venían afirmando que cuando la violencia se mide en actos (frecuencia, diversidad), las mujeres resultan más violentas, pero cuando se mide en heridas, los hombres suelen ser más violentos. Esto, sin embargo, puede estar cambiando, pues cada vez más mujeres asesinan a sus maridos con armas de fuego (en Estados Unidos) o con arma blanca (en España), en un solo acto.

Este escenario nos lleva a pensar ¿por qué para mucha gente es tan difícil aceptar que los varones pueden ser víctimas de sus mujeres? Desde la perspectiva de género, la explicación surge de la adhesión a los estereotipos de género tradicional, en los cuales se piensa que el hombre es siempre fuerte, poderoso, violento y dominador (Montesinos, 2002), y, en contraparte, la mujer se considera sumisa, débil y dependiente, pero se olvida que la violencia es un ejercicio de poder y control, más que de tamaño o fuerza.

Esta construcción social de lo femenino y lo masculino le otorga incuestionablemente una superioridad física y psicológica al varón en diferentes contextos como el familiar, laboral, social y jurídico (Goodrich, Rampage, Ellman \& Halstead, 1989; Rodríguez, Marín \& Leone, 1993), y se conforma como un imperativo que aprisiona a hombres y mujeres en un marco de creencias rígido y paralizante. De ahí que muchas víctimas de ambos géneros se mantengan en silencio en relaciones altamente destructivas en un intento por cumplir con la exigencia social de su "deber ser". 
No obstante, en muchos contextos dichos roles parecen estar sufriendo serias transformaciones. Mujeres de todo el mundo luchan por acceder a vidas más plenas, participando activamente en las diferentes esferas de su vida, mientras que muchos varones buscan deshacerse del papel de "machos" para generar nuevas identidades que les permitan relacionarse consigo mismos y con el mundo de manera diferente (Lomas, 2003). Sin embargo, este optimista panorama se ve opacado por mujeres que repiten aquello de lo que tanto nos quejamos: el recurso de la violencia. $Y$ tal y como ocurrió con las mujeres en las décadas de los cincuenta, sesenta y setenta, ahora la incredulidad es hacia las víctimas masculinas. Quizá por ello existen tan pocos datos al respecto, por lo que urge generar estudios formales que contemplen la prevención, detección, evaluación e intervención en lo individual, pero también la creación de centros e instituciones de apoyo que sensibilicen y protejan a las víctimas masculinas en un nivel macro, como afortunadamente ya ocurre con las mujeres.

\section{Antecedentes}

Según Huertas (2006, citado en Pérez, 2006) cada año mueren más de 1.6 millones de personas en el mundo como consecuencia de actos violentos y más de 500.000 son producto de la violencia interpersonal. En la actualidad, muchos países están preocupados por las muertes que año tras año ocurren entre cónyuges o ex-cónyuges, lo que explica que la violencia doméstica sea considerada un serio problema de salud pública que afecta a un gran número de personas y que atraviesa fronteras raciales, de edad, religión, educación y nivel socioeconómico, lo que genera además enormes demandas de salud, seguridad, asesoría jurídica y asistencial en general.

La definición de CORIAC (1995), que es una de las más incluyentes, reconoce las modalidades de violencia física, sexual, psicológica o emocional, económica o financiera, social y objetal. Sin embargo, se empieza a manejar una más: la violencia legal, que consiste en el abuso de los apoyos legales para ejercer poder, dominio y control sobre la pareja o los hijos.
En este contexto, hoy por hoy existen cada vez más medidas de prevención y erradicación de la violencia (todavía insuficientes), a través de infraestructuras que han ido construyendo recursos al alcance de las manos de miles de víctimas registradas año tras año que, como sabemos, continúan siendo en su mayoría las mujeres. Sin embargo, nos estamos topando con un hecho contundente: las demandas en aumento de varones victimizados a manos de sus mujeres.

Sacar a la luz que los hombres también pueden ser objeto de la violencia doméstica (aunque no necesariamente sean más débiles físicamente, ni ancianos, enfermos o dependientes económicos) ha producido un giro en las investigaciones, escasas aún, a pesar de que autores como Williamson (1995) advirtieron que desde 1975, Murray Straus, Richard Gelles y Susan Steinmetz, pioneros en la investigación sobre violencia doméstica, averiguaron a través de un estudio nacional (en Estados Unidos) que los varones podían conformar el $50 \%$ de las víctimas reales.

Una publicación más reciente de Straus (2006), muestra nada menos que 111 estudios que exponen la alta prevalencia de la violencia bidireccional y cruzada. En palabras del autor:

Durante más de 25 años se han puesto en tela de juicio, a veces con acritud, las investigaciones que demuestran que las mujeres ejercen la violencia física contra sus parejas masculinas en una proporción similar a la ejercida por los varones contra sus parejas femeninas. Sin embargo, los datos de casi 200 estudios son concluyentes (p. 1).

Sacks (2001) agrega que ellas tienen la misma probabilidad de atacar físicamente a sus hombres, en contra del mito de que las mujeres sólo recurren a la violencia por autodefensa. Una explicación a esto es la falta de denuncias por parte de ellos, lo que ha impedido su reconocimiento. Ahora tenemos más datos, y aunque las estadísticas no son del todo claras y los esfuerzos parecen dispersos, asomándonos a lo disponible encontramos señales de alarma. 
Ciertamente, los registros siguen mostrando una mayor violencia en contra de las mujeres (el Instituto Nacional de Justicia y el Centro de Control y Prevención de Enfermedades de Estados Unidos hicieron en el 2005 un cálculo aproximado de $76.5 \%$ de mujeres maltratadas y $23.5 \%$ de varones agredidos), pero la duda estriba en si realmente es así, o es que ellos tienen menor credibilidad social como víctimas $\mathrm{y}$, por tanto, denuncian todavía menos que ellas, y se resisten incluso a acudir a los centros de apoyo, por lo cual estamos hablando de una cifra negra que requiere ser indagada.

Algunos estudios llaman la atención acerca de que mientras la violencia cometida por el varón parece que se ha decrementado en un $6 \%$, la ejercida por la mujer en contra de él ha tenido un incremento del 4\% (Sussman \& Steinmetz, 1998; Trujano, Martínez \& Benítez, 2002). Por otra parte, la Men's Health Network en internet subraya que la violencia es terrible, pero no exclusiva de un género. Al respecto, investigadores como Hoff (1998; 1999) aseguran que en nuestros días, hombres y mujeres abusan del otro casi con la misma frecuencia. Este paulatino deslizamiento en las cifras, en el que las agresiones mutuas y las dirigidas hacia el varón van en aumento, tendrían que hacernos reflexionar acerca de la importancia de trabajar para paliar la violencia, provenga de quien provenga.

Así pues, la mayoría de las aportaciones acerca de la violencia doméstica se han centrado en las víctimas femeninas al ser las más afectadas. Dado que las estadísticas muestran un serio incremento de varones victimizados, resulta importante trabajar con población masculina para intentar arrojar luz a este fenómeno. El objetivo de este trabajo es, entonces, identificar qué actitudes y comportamientos perciben como violentos en su relación de pareja hombres heterosexuales que mantienen una relación de noviazgo y de matrimonio, así como la frecuencia y modalidades con las que se presentan, explorando su posible correlación. Dado lo novedoso del tema, esperamos que esta investigación contribuya a su reconocimiento y apoyo psicosocial.

\section{Método}

\section{Participantes}

Cien hombres que mantenían una relación de pareja no menor a seis meses de duración. Todos residentes en Ciudad de México y presumiblemente pertenecientes a un nivel socioeconómico medio. El rango de edad estaba comprendido entre 18 y 40 años. La distribución de los participantes fue la siguiente:

Muestra 1: 50 hombres en una relación matrimonial no menor a los seis meses de duración.

Muestra 2: 50 hombres en una relación de noviazgo no menor a los seis meses de duración.

\section{Escenario}

Los hombres casados fueron población civil contactada azarosamente en el Distrito Federal. Los que se encontraban en una relación de noviazgo eran estudiantes de licenciatura de la Universidad Nacional Autónoma de México, Campus Iztacala.

\section{Instrumento}

Se utilizó el VIDOFyP (Violencia Doméstica: Frecuencia y Percepción, 1998, en Trujano \& Mendoza, 2003), que permite identificar la percepción y frecuencia de episodios violentos en la pareja. Se adaptaron los reactivos 4, 18, 22, 26 y 29 para la relación de noviazgo de la siguiente manera:

4) Mi pareja cuestiona todo el tiempo la forma en que dispongo de mi dinero.

18) Mi pareja decide sin mí, cuánto y en qué gastar cuando salimos juntos.

22) Mi pareja me critica si no hago las cosas cuándo y cómo lo desea.

26) Mi pareja decide sin mí cómo distribuir su dinero, pero interfiere en cómo lo hago yo. 
29) Mi pareja no me informa cuánto gana o tiene, pero me presiona para saber cuánto tengo yo.

El inventario consta de 30 reactivos que se responden a través de una escala Lickert, en los cuales se describen conductas y actitudes que expresan seis formas de violencia comúnmente ejercidas en relaciones de pareja abusivas, como la física, psicológica, objetal, económica, social y sexual. Los reactivos se distribuyen de la siguiente manera: Violencia física: 17, 19, 21 y 23. Violencia psicológica: 2, 3, 5, 7, 9, 14, 16 y 22 . Violencia social: $1,6,8,10,12$ y 15 . Violencia sexual: 11 , 13, 24, 27 y 30. Violencia económica: 4, 18, 26 y 29. Violencia objetal: 20,25 y 28

\section{Procedimiento}

Antes de comenzar con la aplicación del instrumento, se le proporcionó a cada participante un consentimiento informado en el que se le explicó en qué consistía la investigación, se le solicitó su participación anónima y voluntaria, y se le informó que podía retirarse en el momento que lo deseara. Se mantuvo siempre un trato respetuoso, ético y profesional y se les instruyó para responder al inventario. El tiempo aproximado de respuesta fue de quince minutos.

\section{Resultados}

De acuerdo con el análisis estadístico, se pudo observar que, en general, en las dos muestras tanto la frecuencia como la percepción de actitudes y comportamientos violentos de la pareja fueron bajas si las comparamos con los puntajes máximos que podían obtenerse en las escalas que era de 150 puntos.

Con respecto a la frecuencia obtuvimos las medias ( $X=47.02$ para la muestra $1 ; X=40.80$ para la muestra 2), sus medianas (44 y 38.5 ) y sus modas (33 y 47). En cuanto a la percepción las medias fueron (73.54 y 59.8$)$, sus medianas (60.5 y 37.5$)$ y modas (32 y 30 ). Esto apunta a que en las relaciones de pareja de ambas muestras son poco frecuentes los episodios violentos, pero también existe una baja percepción de los mismos.
Sin embargo, estos datos también indican que los episodios violentos presentan una mayor incidencia y percepción en los hombres casados que en los que mantienen una relación de noviazgo. Al aplicar una $\mathrm{t}$ de Student para muestras independientes encontramos que sí existen diferencias significativas en cuanto a la escala de frecuencia, es decir, los hombres casados reportan una mayor incidencia de episodios violentos que los hombres que mantienen una relación de noviazgo $(\mathrm{t}=2.91, \mathrm{p}<.005)$, mientras que en la escala de percepción las diferencias no resultaron significativas $(\mathrm{t}=1.834)$.

Por otra parte, para realizar una comparación entre la escala de frecuencia y percepción, se aplicó una prueba $t$ de Student para muestras relacio-

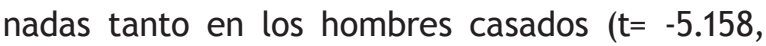
$\mathrm{p}<.05)$ como en los que mantienen una relación de noviazgo ( $t=-3.652, p<.05$ ), y se obtuvieron en cada caso diferencias significativas entre la frecuencia y percepción, es decir, en ambos casos la percepción es mayor que la frecuencia.

Con el propósito de saber si existía relación entre la escala de frecuencia y percepción de las conductas violentas, se obtuvieron coeficientes de correlación rho de Spearman. Se encontró en el caso de la muestra de hombres casados un coeficiente de .320 con una $p<.05$ y en la muestra de hombres con una relación de noviazgo un coeficiente de .423 con una $\mathrm{p}<.05$. De acuerdo con los valores obtenidos, podemos afirmar que en la muestra de hombres con una relación de noviazgo, la relación entre frecuencia y percepción resultó ser positiva moderada, mientras que en la muestra de hombres casados la correlación resultó ser positiva débil, es decir, a mayor percepción de episodios violentos mayor frecuencia de éstos. En ambos casos las correlaciones resultaron significativas.

Se realizó posteriormente otro análisis para observar la escala de frecuencia y percepción en la relación de pareja de los hombres de cada muestra, pero en esta ocasión por modalidades, es decir, por los tipos de violencia que evalúa el instrumento: 


\section{Escala de Frecuencia}

En general, las puntuaciones obtenidas en cada una de las modalidades de violencia, tanto en la muestra de los hombres casados como en la de los hombres con una relación de noviazgo, fueron bajas al compararlas con los puntajes mínimos y máximos que podían obtenerse en cada una de dichas modalidades, lo que significa que se presentan poco en sus relaciones, aunque son más frecuentes en los casados. En ambas muestras las modalidades de violencia psicológica, social y sexual resultaron las más frecuentes. Las de menor incidencia fueron la objetal, la física y la económica. A continuación se describen los valores obtenidos (Tabla 1).

Tabla 1. Medidas descriptivas por muestra, de la escala de frecuencia por modalidades de violencia en la relación de pareja

\begin{tabular}{|c|c|c|c|c|c|c|}
\hline & $\frac{\pi}{\pi}$ &  & $\begin{array}{l}\frac{\pi}{2} \\
\frac{0}{2}\end{array}$ & 峁 & $\dot{\sum}$ & $\underset{⿱ 亠 凶}{\stackrel{x}{\Sigma}}$ \\
\hline \multicolumn{7}{|l|}{ Matrimonio } \\
\hline \multicolumn{7}{|l|}{$n=50$} \\
\hline Psicológica & 14.56 & 13.50 & 10 & 5.29 & 8 & 40 \\
\hline Física & 5.54 & 4 & 4 & 2.18 & 4 & 20 \\
\hline Sexual & 7.04 & 7 & 5 & 2.08 & 5 & 25 \\
\hline Económica & 6.80 & 6 & 4 & 2.44 & 4 & 20 \\
\hline Social & 9.42 & 8.5 & 8 & 3.06 & 6 & 30 \\
\hline Objetal & 3.66 & 3 & 3 & 1.27 & 3 & 15 \\
\hline \multicolumn{7}{|l|}{ Noviazgo } \\
\hline \multicolumn{7}{|l|}{$n=50$} \\
\hline Psicológica & 12.86 & 12 & 12 & 3.68 & 8 & 40 \\
\hline Física & 5.08 & 4 & 4 & 1.76 & 4 & 20 \\
\hline Sexual & 6.48 & 5 & 5 & 2.33 & 5 & 25 \\
\hline Económica & 4.48 & 4 & 4 & 1.01 & 4 & 20 \\
\hline Social & 8.54 & 8 & 8 & 2.53 & 6 & 30 \\
\hline Objetal & 3.36 & 3 & 3 & .85 & 3 & 15 \\
\hline
\end{tabular}

\section{Escala de Percepción}

La Tabla 2 presenta las puntuaciones promedio en ambas muestras, así como también los puntajes mínimos y máximos que podían obtenerse. Se observaron valores bajos, pero los hombres casados mostraron nuevamente una mayor percepción de cada una de las modalidades de violencia. Otra vez, en las dos muestras las modalidades de violencia psicológica, social y sexual resultaron ser las mejor percibidas, mientras que la objetal, la física y la económica fueron las menos percibidas. Se pueden observar los valores obtenidos en la Tabla 2.

Tabla 2. Medidas descriptivas de la escala de percepción por modalidades de violencia en la relación de pareja por muestra

\begin{tabular}{|c|c|c|c|c|c|c|}
\hline & $\begin{array}{l}\frac{\pi}{\pi} \\
\frac{\pi}{2} \\
\end{array}$ & $\stackrel{D}{\Sigma}^{\infty}$ & $\frac{\pi}{0}$ & $\dot{\vec{n}}$ & $\dot{\Sigma}$ & 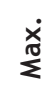 \\
\hline \multicolumn{7}{|l|}{$\begin{array}{l}\text { Matrimonio } \\
\mathrm{n}=50\end{array}$} \\
\hline Psicológica & 19.06 & 17 & 17 & 9.03 & 8 & 40 \\
\hline Fisica & 11.38 & 10.50 & 4 & 6.50 & 4 & 20 \\
\hline $\begin{array}{l}\text { Sexual } \\
\text { Fconómica }\end{array}$ & 12.92 & 12 & 5 & 7.06 & 5 & 25 \\
\hline Social & 8.34 & 7.50 & 4 & 4.22 & 4 & 20 \\
\hline Objetal & 13.76 & 11.50 & 7 & 7.08 & 6 & 30 \\
\hline & 8.08 & 6 & 3 & 4.97 & 3 & 15 \\
\hline \multicolumn{7}{|l|}{$\begin{array}{l}\text { Noviazgo } \\
\mathrm{n}=50\end{array}$} \\
\hline Psicológica & 16.04 & 11 & 8 & 9.14 & 8 & 40 \\
\hline Física & 9.38 & 5.50 & 4 & 6.74 & 4 & 20 \\
\hline Sexual & 9.78 & 5 & 5 & 6.78 & 5 & 25 \\
\hline Económica & 6.98 & 4 & 4 & 4.70 & 4 & 20 \\
\hline Social & 11.36 & 8 & 6 & 7.13 & 6 & 30 \\
\hline Objetal & 6.26 & 3 & 3 & 4.38 & 3 & 15 \\
\hline
\end{tabular}

Para conocer si existen diferencias entre la escala de frecuencia y percepción de la violencia en cada una de las muestras, se aplicó una t de Student para grupos independientes, como resultado se encontró que en la escala de frecuencia de la violencia económica sí hubo diferencias significativas entre los hombres casados y los que mantienen una relación de noviazgo, es decir, que esta modalidad de violencia se presenta con mayor frecuencia en los hombres casados. Mientras que en las modalidades de violencia psicológica, física, sexual, social y objetal no se encontraron diferencias significativas en cuanto a la escala de frecuencia entre ambas muestras (Tabla 3).

En cuanto a la escala de percepción de la violencia sexual sí se encontraron diferencias significa- 
tivas, es decir, que esta modalidad de violencia tiene una mayor percepción en la muestra de hombres casados. Mientras que en las modalidades de violencia psicológica, física, económica, social y objetal no se encontraron diferencias significativas entre las muestras (Tabla 3).

Tabla 3. Comparación entre la muestra de hombres casados y en relación de noviazgo de la escala de frecuencia y percepción de las modalidades de violencia

\begin{tabular}{|c|c|c|c|c|c|}
\hline & \multirow{2}{*}{$\begin{array}{l}\text { Tipo de } \\
\text { relación }\end{array}$} & \multicolumn{2}{|c|}{ Frecuencia } & \multicolumn{2}{|c|}{ Percepción } \\
\hline & & $\begin{array}{c}\mathrm{t} \text { de } \\
\text { Student }\end{array}$ & Prob. & $\begin{array}{c}\mathrm{t} d \mathrm{de} \\
\text { Student }\end{array}$ & Prob. \\
\hline Psicológica & $\begin{array}{l}\text { Matrimonio } \\
\text { y noviazgo }\end{array}$ & 1.865 & .066 & 1.662 & .100 \\
\hline Física & $\begin{array}{l}\text { Matrimonio } \\
\text { y noviazgo }\end{array}$ & 1.162 & .248 & 1.510 & .134 \\
\hline Sexual & $\begin{array}{l}\text { Matrimonio } \\
\text { y noviazgo }\end{array}$ & 1.267 & .208 & 2.268 & .026 \\
\hline Económica & $\begin{array}{l}\text { Matrimonio } \\
\text { y noviazgo }\end{array}$ & 6.205 & .000 & 1.523 & .131 \\
\hline Social & $\begin{array}{l}\text { Matrimonio } \\
\text { y noviazgo }\end{array}$ & 1.567 & .120 & 1.689 & .094 \\
\hline Objetal & $\begin{array}{l}\text { Matrimonio } \\
\text { y noviazgo }\end{array}$ & 1.386 & .169 & 1.941 & .055 \\
\hline
\end{tabular}

Al realizar una comparación en cada muestra sobre la escala de frecuencia y percepción de cada tipo de violencia, se aplicó una t de Student para grupos relacionados, y se obtuvieron valores en cada muestra que indican que en todas las modalidades de violencia existen diferencias significativas entre éstas, siendo mayor la percepción que la frecuencia (Tabla 4).

Tabla 4. Comparación entre la escala de frecuencia y percepción por muestra

\begin{tabular}{lcccc}
\hline & \multicolumn{2}{c}{$\begin{array}{c}\text { Matrimonio } \\
\mathrm{n}=50\end{array}$} & \multicolumn{2}{c}{$\begin{array}{c}\text { Noviazgo } \\
\mathrm{n}=50\end{array}$} \\
\hline & $\begin{array}{c}\mathrm{t} \text { de } \\
\text { Student }\end{array}$ & Prob. & $\begin{array}{c}\mathrm{t} \text { de } \\
\text { Student }\end{array}$ & Prob. \\
\hline $\begin{array}{l}\text { Psicológica } \\
\text { Frecuencia }\end{array}$ & & & & \\
$\begin{array}{l}\text { Percepción } \\
\text { Física }\end{array}$ & -3.445 & .001 & -2.534 & .015 \\
$\begin{array}{l}\text { Frecuencia } \\
\text { Percepción }\end{array}$ & -6.443 & .000 & -4.416 & .000 \\
\hline
\end{tabular}

\begin{tabular}{lcccc}
\hline & \multicolumn{2}{c}{$\begin{array}{c}\text { Matrimonio } \\
\mathrm{n}=50\end{array}$} & \multicolumn{2}{c}{$\begin{array}{c}\text { Noviazgo } \\
\mathrm{n}=50\end{array}$} \\
\hline & $\begin{array}{c}\mathrm{t} \text { de } \\
\text { Student }\end{array}$ & Prob. & $\begin{array}{c}\mathrm{t} \text { de } \\
\text { Student }\end{array}$ & Prob. \\
\hline $\begin{array}{c}\text { Sexual } \\
\text { Frecuencia }\end{array}$ & & & & \\
$\begin{array}{c}\text { Percepción } \\
\text { Económica }\end{array}$ & -5.655 & .000 & -3.476 & .001 \\
$\begin{array}{c}\text { Frecuencia } \\
\text { Percepción }\end{array}$ & -2.681 & .010 & -3.655 & .001 \\
$\begin{array}{c}\text { Social } \\
\text { Frecuencia }\end{array}$ & & & & \\
$\begin{array}{c}\text { Percepción } \\
\text { Objetal }\end{array}$ & -4.222 & .000 & -2.822 & .007 \\
$\begin{array}{c}\text { Frecuencia } \\
\text { Percepción }\end{array}$ & -6.153 & .000 & -4.721 & .000 \\
\hline
\end{tabular}

Por último, al explorar la relación entre la escala de percepción y frecuencia de cada una de las modalidades de violencia para cada muestra, a través de una rho de Spearman, se produjeron los siguientes hallazgos (Tabla 5):

Psicológica: en esta modalidad de violencia se encontró que la muestra de hombres con una relación de noviazgo (rho = .498, $\mathrm{p}<.05$ ) obtuvo una correlación positiva moderada, mientras que la muestra de hombres casados (rho $=.303, \mathrm{p}<.05$ ) obtuvo una correlación positiva débil. La correlación indica que a mayor percepción mayor frecuencia de episodios violentos.

Física: en esta forma se observó que en ambas muestras, tanto en la de hombres casados (rho $=.313, \mathrm{p}<.05$ ) como en la de hombres con una relación de noviazgo (rho =.268, p>.060), la correlación resultó ser positiva débil. En la muestra de hombres con una relación de noviazgo la correlación no fue significativa.

Sexual: en ésta obtuvimos en la muestra de hombres casados ( $\mathrm{rho}=.121, \mathrm{p}>$.404) una correlación positiva débil, mientras que en la muestra de hombres con una relación de noviazgo (rho $=.437$, $\mathrm{p}<.05)$ se encontró una correlación positiva moderada. La correlación no fue significativa en el caso de los hombres casados.

Económica: en esta modalidad se encontró en la muestra de hombres casados ( $r$ ho $=.326, \mathrm{p}<.05)$ una 
correlación positiva débil. Mientras que en la muestra de hombres con una relación de noviazgo ( $r$ ho= $-.022, p>.05$ ) notamos una correlación casi nula.

Social: en esta opción observamos que en ambas muestras, tanto en la de hombres casados (rho= $.210, p>.05$ ) como en la de hombres con una relación de noviazgo (rho $=.330, \mathrm{p}<.05$ ), la correlación resultó ser positiva débil. En la muestra de hombres casados la correlación no fue significativa.

Objetal: nuevamente en ambas muestras, tanto en la de hombres casados ( $r h o=.182, p>.05$ ) como en la de hombres con una relación de noviazgo (rho $=.332, \mathrm{p}<.05)$, la correlación resultó ser positiva débil. En la muestra de hombres casados la correlación no fue significativa.

Tabla 5. Correlación entre la escala de frecuencia y percepción por modalidad de violencia en cada muestra

\begin{tabular}{lcccc}
\hline & \multicolumn{2}{c}{$\begin{array}{c}\text { Matrimonio } \\
\mathrm{n}=50\end{array}$} & \multicolumn{2}{c}{$\begin{array}{c}\text { Noviazgo } \\
\mathrm{n}=50\end{array}$} \\
& $\begin{array}{c}\text { rho de } \\
\text { Spearman's }\end{array}$ & Prob. & $\begin{array}{c}\text { rho de } \\
\text { Spearman's }\end{array}$ & Prob. \\
\hline $\begin{array}{c}\text { Psicológica } \\
\text { Frecuencia } \\
\text { Percepción }\end{array}$ & .303 & .032 & .498 & .000 \\
$\begin{array}{l}\text { Física } \\
\text { Frecuencia }\end{array}$ & & & & \\
$\begin{array}{l}\text { Percepción } \\
\text { Sexual }\end{array}$ & .313 & .027 & .268 & .060 \\
$\begin{array}{l}\text { Frecuencia } \\
\text { Percepción }\end{array}$ & .121 & .404 & .437 & .001 \\
$\begin{array}{l}\text { Económica } \\
\text { Frecuencia }\end{array}$ & & & & \\
Percepción & .326 & .021 & -.022 & .879 \\
$\begin{array}{c}\text { Social } \\
\text { Frecuencia } \\
\text { Percepción }\end{array}$ & .210 & .143 & .330 & .019 \\
Objetal & & & & \\
$\begin{array}{l}\text { Frecuencia } \\
\text { Percepción }\end{array}$ & .182 & .206 & .332 & .019 \\
\hline
\end{tabular}

Para comparar la escala de frecuencia de las modalidades de violencia entre sí, se aplicó una $t$ de Student para muestras relacionadas. El mismo análisis se llevó a cabo para comparar la percepción. Los resultados pueden apreciarse en las tablas 6 y 7.
Tabla 6. Comparación de las escalas de frecuencia de las modalidades de violencia por muestra

\begin{tabular}{|c|c|c|c|c|}
\hline & \multicolumn{2}{|c|}{$\begin{array}{c}\text { Matrimonio } \\
n=50\end{array}$} & \multicolumn{2}{|c|}{$\begin{array}{c}\text { Noviazgo } \\
n=50\end{array}$} \\
\hline & $\begin{array}{c}\text { t de } \\
\text { student }\end{array}$ & Prob. & $\begin{array}{c}\mathrm{t} \text { de } \\
\text { student }\end{array}$ & Prob. \\
\hline $\begin{array}{l}\text { Física } \\
\text { comparada con: } \\
\text { Sexual } \\
\text { Objetal } \\
\text { Económica } \\
\text { Social } \\
\text { Psicológica }\end{array}$ & $\begin{array}{l}-5.324 \\
7.152 \\
-3.627 \\
-9.940 \\
-12.767\end{array}$ & $\begin{array}{l}.000 \\
.000 \\
.001 \\
.000 \\
.000\end{array}$ & $\begin{array}{c}-3.045 \\
6.490 \\
2.189 \\
-10.737 \\
-15.335\end{array}$ & $\begin{array}{l}.004 \\
.000 \\
.033 \\
.000 \\
.000\end{array}$ \\
\hline $\begin{array}{l}\text { Sexual } \\
\text { comparada con: } \\
\text { Objetal } \\
\text { Económica } \\
\text { Social } \\
\text { Psicológica }\end{array}$ & $\begin{array}{l}13.491 \\
.706 \\
-5.555 \\
-11.272\end{array}$ & $\begin{array}{l}.000 \\
.484 \\
.000 \\
.000\end{array}$ & $\begin{array}{c}9.781 \\
5.959 \\
-4.612 \\
-14 . .240\end{array}$ & $\begin{array}{l}.000 \\
.000 \\
.000 \\
.000\end{array}$ \\
\hline $\begin{array}{l}\text { Objetal } \\
\text { comparada con: } \\
\text { Psicológica } \\
\text { Económica } \\
\text { Social }\end{array}$ & $\begin{array}{l}-15.481 \\
-9.638 \\
-14.095\end{array}$ & $\begin{array}{l}.000 \\
.000 \\
.000\end{array}$ & $\begin{array}{l}-19.933 \\
-6.226 \\
-15.622\end{array}$ & $\begin{array}{l}.000 \\
.000 \\
.000\end{array}$ \\
\hline $\begin{array}{l}\text { Económica } \\
\text { comparada con: } \\
\text { Social } \\
\text { Psicológica }\end{array}$ & $\begin{array}{c}-7.566 \\
-12.838\end{array}$ & $\begin{array}{l}.000 \\
.000\end{array}$ & $\begin{array}{l}-11.362 \\
-16.148\end{array}$ & $\begin{array}{l}.000 \\
.000\end{array}$ \\
\hline $\begin{array}{l}\text { Psicológica } \\
\text { comparada con: } \\
\text { Social }\end{array}$ & 9.548 & .000 & 10.473 & .000 \\
\hline
\end{tabular}

Tabla 7. Comparación de las escalas de percepción de las modalidades de violencia por muestra

\begin{tabular}{lcccc}
\hline & \multicolumn{2}{c}{$\begin{array}{c}\text { Matrimonio } \\
\mathrm{n}=50\end{array}$} & \multicolumn{2}{c}{$\begin{array}{c}\text { Noviazgo } \\
\mathrm{n}=50\end{array}$} \\
\cline { 2 - 5 } & $\begin{array}{c}\text { t de } \\
\text { Student }\end{array}$ & Prob. & $\begin{array}{c}\text { t de } \\
\text { Student }\end{array}$ & Prob. \\
\hline Física comparada & & & & \\
con: & -4.049 & .000 & -1.052 & .298 \\
Sexual & 9.096 & .000 & 8.036 & .000 \\
Objetal & 6.405 & .000 & 5.400 & .000 \\
Económica & -4.732 & .000 & -5.954 & .000 \\
Social & -12.251 & .000 & -12.208 & .000 \\
Psicológica & & & & \\
Sexual compara- & & & & \\
da con: & 11.517 & .000 & 8.067 & .000 \\
Objetal & 8.718 & .000 & 6.113 & .000 \\
Económica & -2.022 & .049 & -3.871 & .000 \\
Social & -10.745 & .000 & -9.713 & .000 \\
Psicológica & & & & \\
\hline
\end{tabular}




\begin{tabular}{lcccc}
\hline & \multicolumn{2}{c}{$\begin{array}{c}\text { Matrimonio } \\
\mathrm{n}=50\end{array}$} & \multicolumn{2}{c}{$\begin{array}{c}\text { Noviazgo } \\
\mathrm{n}=50\end{array}$} \\
\cline { 2 - 5 } & $\begin{array}{c}\mathrm{t} \text { de } \\
\text { Student }\end{array}$ & Prob. & $\begin{array}{c}\mathrm{t} \text { de } \\
\text { Student }\end{array}$ & Prob. \\
& & & & \\
\hline $\begin{array}{l}\text { Objetal compara- } \\
\text { da con: }\end{array}$ & -15.415 & .000 & -12.513 & .000 \\
$\begin{array}{l}\text { Psicológica } \\
\text { Económica }\end{array}$ & -.761 & .450 & -3.078 & .003 \\
$\begin{array}{l}\text { Social } \\
\text { Económica }\end{array}$ & -12.066 & .000 & -10.477 & .000 \\
parada con: & & & & \\
$\begin{array}{l}\text { Social } \\
\text { Psicológica }\end{array}$ & -10.197 & .000 & -9.698 & .000 \\
$\begin{array}{l}\text { Psicológica } \\
\text { parada con: }\end{array}$ & -13.142 & .000 & -12.405 & .000 \\
Social & & & & \\
\hline
\end{tabular}

\section{Discusión}

Dado que los estudios sobre violencia doméstica en contra de los varones son escasos, resulta difícil tener puntos de referencia con los cuales contrastar nuestros hallazgos. Por otro lado, debemos recordar que trabajamos con una muestra pequeña y, además, que los varones en relaciones de noviazgo fueron población universitaria, lo que restringe las posibilidades de generalización de nuestros hallazgos.

Sin embargo, podemos decir que los resultados de esta investigación apuntan a que aún durante el noviazgo, pero más durante la relación de matrimonio, pueden ocurrir comportamientos violentos, y aunque la incidencia en nuestras muestras fue baja, no deja de ser un indicador preocupante, pues de acuerdo con Zubizarreta, Sarasúa, Echeburúa, de Corral, Sauca y Emparanza (1994), una vez que se han presentado episodios de violencia, es probable que aumenten tanto en frecuencia como en intensidad, reduciendo las probabilidades y los recursos de la víctima para salir de la relación.

También se obtuvieron niveles bajos de percepción, lo que puede traducirse en que la violencia puede ser minimizada como conflictos personales de la compañera o simplemente como desavenencias propias de cualquier pareja. Por otro lado, el apego a los roles de género tradicional puede llevar a los varones a la negación de lo que está su- cediendo. Algunos testimonios en los consultorios muestran que muchos de ellos manifiestan tener "problemas" con su compañera, que "cada vez se salen más de control", en un intento por justificar lo que está ocurriendo, y es durante el transcurso de la intervención terapéutica que aceptan ser víctimas de la violencia de sus mujeres. Ramírez (2002) menciona al respecto la dificultad e incluso el temor de muchos varones por no cumplir con el papel socialmente asignado.

Por lo mismo, parece conveniente incluir, en este tipo de estudios, entrevistas que personalicen la obtención de información y que eviten, en alguna medida, que los varones lleguen a contestar pensando más en buscar respuestas correctas o en dar una "imagen adecuada", si bien cabe señalar que el instrumento utilizado ha sido probado en estudios anteriores que han mostrado su validez y confiabilidad (Trujano \& Mata, 2002; Trujano \& Mendoza, 2003; Trujano, Nava, Tejeda \& Gutiérrez, 2006).

Otro hallazgo fue que los hombres casados tuvieron niveles más altos tanto de frecuencia como de percepción de violencia. Esto es acorde con lo señalado por autores como Beck (1993), quien establece que la vida conyugal trae consigo una serie de deberes, derechos y obligaciones en la convivencia diaria que aumentan la probabilidad de afrontar situaciones de conflicto en cuanto a distribución de dinero, crianza de los hijos, manejo del tiempo libre, etcétera. Mientras que en el noviazgo la idealización del amor y las expectativas de futuro pueden llevar a hacer invisibles las diferencias (Trujano y Mata, 2002; Hanson, 2005).

De cualquier modo, esta etapa de la vida merece ser más explorada, ahora con varones, especialmente porque los estudios apuntan a que muchas relaciones violentas lo fueron desde el noviazgo. En México, por ejemplo, el Instituto Nacional de las Mujeres mostró en el 2004 que nueve de cada diez mujeres habían sufrido violencia en el noviazgo, lo cual fue consistente con lo publicado por el gobierno del Distrito Federal al realizar el programa Amor es... sin violencia.

Por otro lado, se encontró también y de manera significativa, que en ambas muestras la percep- 
ción de episodios violentos fue mayor que su frecuencia, lo que pudo deberse a que actualmente existe mucha información en torno al tema de la violencia (si bien está dirigida a las mujeres), lo que quizá facilitó su identificación. Estos resultados son consistentes con los de Trujano y Mata (2002) quienes también encontraron que la sola discriminación de los episodios violentos no fue suficiente para evitarlos, como sugirieron Trujano y Mendoza en el 2003. De hecho, se observó en ambas muestras una correlación positiva y significativa entre la percepción y la frecuencia, lo que significa que a mayor percepción, mayor presencia de episodios violentos.

Esto quiere decir, por un lado, que no es suficiente identificar estos comportamientos, sino que es necesario proveer herramientas y habilidades específicas de afrontamiento, y, por el otro, que indudablemente en muchos contextos se está brindando educación a los hombres de manera diferente a la tradicional, es decir, se les está inculcando el respeto que deben tener hacia las mujeres (Fiebert, 2004), lo que puede explicar que muchos varones aunque perciban que los comportamientos de su mujer son violentos, los toleren y aprendan a vivir con ellos.

En cuanto a las modalidades de violencia, se encontró para ambas muestras que la psicológica, la social y la sexual presentaron una mayor percepción y también una mayor frecuencia, mientras que la económica, la física y la objetal tuvieron los puntajes más bajos. Ya antes se ha argumentado que la violencia psicológica y la social parecen ser más recurrentes debido a la facilidad con que pueden emplearse y a que no dejan huellas visibles. En cuanto a la sexual, es factible que la transformación en los roles de género esté facilitando actitudes en las mujeres antes impensables, incluido llegar al sometimiento y control de la sexualidad en la relación con su pareja.

El hallazgo de que la violencia económica es mayor entre los casados parece lógico dado que en la actualidad cada vez más mujeres salen del ámbito doméstico para incorporarse al laboral, lo que les permite contar con sus propios recursos; otras, de hecho, se han convertido en las principales aportadoras al ingreso familiar; existen también amas de casa que administran el salario del marido. En cualquier caso, es más factible este tipo de violencia entre cónyuges que entre novios, quienes suelen manejar su dinero de manera independiente.

Resta señalar que la modalidad de violencia sexual resultó mejor percibida por los casados, lo que se explica a través de que los novios pueden traducirla como algo excitante y novedoso en su relación, mientras que los casados pueden percibirla como transgresión a los límites dentro de los cuales se movía la pareja, y como una agresión a su intimidad y a su masculinidad.

Para terminar, es importante subrayar la importancia de trabajar con los jóvenes como una tarea impostergable, teniendo en cuenta que el noviazgo puede ser la antesala de la violencia conyugal. Un estudio comparativo de género realizado recientemente por Corral y Calvete (2006) con jóvenes universitarios de entre 18 y 30 años mostró que, en cuanto a la agresión psicológica moderada, hubo más casos de mujeres que relataron haber recibido y utilizado ésta, mientras que para la violencia física ejercida, un mayor porcentaje de mujeres (en comparación con los varones), reconoció haberla empleado en contra de su pareja. En cuanto a coerción sexual, el patrón se invirtió. Lo anterior nos muestra que incluso en parejas muy jóvenes la violencia está empezando a constituirse como un estilo normalizado de afrontar los conflictos. De hecho, la violencia cada vez más deja de ser una característica exclusivamente masculina, como muestra la crónica de Tereixa Constenla (2007), quien apuntó que las jóvenes empiezan a recurrir a comportamientos agresivos y a cometer delitos violentos antes impensables en mujeres, lo que constató en el Centro de Reforma Juvenil de Cádiz, España, en donde la mayoría de las menores ingresaron por maltrato familiar, lesiones y agresiones, tendencia ratificada por algunas fiscalías que asisten a un incremento de delitos protagonizados por chicas. 
De esta investigación se desprende la imperiosa necesidad de generar esfuerzos dirigidos a prevenir la violencia en la pareja, no sólo involucrando a ambos protagonistas, como emisores y receptores, sino también evitando la perpetuación de roles tanto de víctima como de victimario/a. Infortunadamente, la realidad es que la mayor parte de este trabajo se realiza con víctimas que llegan a hospitales, centros de asistencia y ministerios públicos después de un encuentro grave con su pareja (Saltijeral, Ramos \& Caballero, 1998).

La prevención puede insertarse en un nivel primario tendiente a evitar que los episodios violentos ocurran. Por ejemplo, trabajando con niños y adolescentes a través de proveer información basada en la equidad y el respeto, así como con jóvenes que pueden estar en el proceso de elección de una pareja. Un nivel secundario tendría que detectar (especialmente en escuelas e institutos) personas y situaciones de riesgo y proveerlas además de habilidades muy específicas, mediante la creación de repertorios de interacción social justos y eficaces. Un tercer nivel estaría dirigido a quienes ya cuenten con episodios violentos en sus relaciones de pareja, involucrando sus características y contextos particulares, pero enfatizando en el análisis y la reflexión de sí mismos y de su actuación como parejas, en aras de alejarlos de los convencionalismos impuestos socialmente y colocándolos en la posibilidad de involucrarse en proyectos de vida equitativos, pues, infortunadamente, como dice Callirgos (2003) "nos relacionamos con nosotros mismos, y con nuestros roles, según concepciones y valoraciones culturales" (p. 59).

Por lo mismo, el telón de fondo tendría que ser el cuestionamiento de la adhesión a roles de género tradicionales (hombre/violencia, mujer/sumisión) o novedosos (mujer/violencia, varón/víctima), atravesados por una visión dicotómica que impide ver toda la gama de posibilidades. Esto lleva a la falsa creencia de que las personas deben elegir ser víctimas o verdugos (como si no hubiera más opciones) y, por lo mismo, deciden lo segundo, lo que facilita tener el poder y el dominio sobre los demás, lo que de paso obstaculiza la derivación de nuevas concepciones y actitudes que favorezcan relaciones más igualitarias.

\section{Conclusiones}

La violencia doméstica ejercida por las mujeres en contra de los varones es hoy por hoy una realidad, existe, y debemos reaccionar frente a ello. Ciertamente, los registros indican una muy superior cifra de mujeres víctimas, lo que es terrible y vergonzoso, cosa que nadie puede negar, pero el ser menos numéricamente no debería significar importar menos.

Cualquier víctima de la violencia merece atención, respeto y apoyo. Su sufrimiento y su dolor son igualmente legítimos, se trate de una mujer o de un hombre, de un niño/a o un anciano/a. Esgrimir que son pocos los varones victimizados es recurrir al argumento que tantas veces rebatimos las mujeres durante todos estos años: ¿y las cifras negras?, ¿y las dificultades para denunciar?, ¿y la falta de credibilidad?, ¿y las burlas, los malos tratos y segundas victimizaciones por parte de las instancias que deberían protegernos?, ¿y el miedo a las represalias, a perder a la pareja, a separar a la familia?

El tiempo demostró que las víctimas femeninas no eran pocas, sino que sólo alcanzábamos a ver la punta del iceberg. Igual que sucedió con nosotras, hoy cada vez más varones se atreven a romper el silencio y a denunciar rompiendo tabúes y enfrentando a una sociedad que parece repetir la historia: "son casos aislados... son las excepciones". Argumentar con mitos como que ellos suelen ser más grandes y más fuertes, o que si alguna mujer violenta a su marido es siempre en defensa propia, o que ellos hacen cosas que provocan el enojo de sus mujeres es rizar el rizo, es decir, complicar las cosas más de lo necesario. Es no tener memoria. Es borrar de un plumazo años de valiosas luchas feministas en pro de la equidad.

Cuando les negamos a las víctimas varones sus derechos los estamos discriminando por su género. Estamos olvidando que la violencia no es natural (sino aprendida), que es dirigida e intencional, y que tiene que ver con poder, con abuso y con control. Ponerle apellido masculino al ejercicio de la violencia y rostro femenino al papel de víctima es encorsetar, es perpetuar los roles tradicionales, y negar o justificar la violencia femenina equivale a ser su cómplice, a legitimarla. 
Autores tan reconocidos como Seidler (2003) subrayan que la teoría feminista temprana insistió en identificar la masculinidad como una relación exclusivamente de poder, como si no hubiera manera de $<<$ redescubrirla $>$, ya que el objetivo era deconstruirla "[...] era como si los hombres no tuvieran ninguna oportunidad para el cambio o ningún modo que permitiera la redención de la masculinidad" (p. 207). Por ello, el feminismo tradicional iba unido a la proclamación de igualdad de derechos.

La segunda oleada del feminismo extendió una importante premisa "lo personal es político", lo que significaba que el poder debió ser analizado dentro de la esfera personal de las relaciones íntimas, al igual que dentro de la esfera pública de la política (Seidler, 2003). Reconocer que las estructuras sociales de poder se reproducen en casa fue un avance, negar que los protagonistas se estén invirtiendo es un retroceso.

Es imprescindible subrayar que la perspectiva de género implica que, precisamente por ser determinadas social e históricamente, las relaciones de inequidad, dominación, discriminación y violencia entre hombres y mujeres pueden y deben cambiar. De ahí la importancia de aceptar la multiplicidad de formas de ser masculinas, como hemos reivindicado el derecho a la pluralidad de formas de ser femeninas. Construir nuevos vínculos a partir de las diferencias abrirá posibilidades de complementación, de búsqueda de alternativas y de relaciones con sentido de equidad entre hombres y mujeres.

Urge rebasar los clichés de género y asumir que lo importante es luchar en contra de la violencia, provenga de quien provenga. Burlarse de los hombres, ridiculizarlos, someterlos o violentarlos no sólo no favorece la igualdad, sino que transfiere la hegemonía masculina a las mujeres. "La violencia, finalmente, forma parte de un sistema de dominación, pero es al mismo tiempo una medida de su imperfección" (Connell, 2003, p. 48). Debemos trabajar por una mayor justicia entre los géneros sin sometedores(as) ni sometidos(as), en escenarios dibujados por el respeto a la diversidad que nos guíe a la equidad en el contexto de una sociedad más democrática y libre.

\section{Referencias}

Beck, A. (1993). Con el amor no basta. Cómo superar malentendidos, resolver conflictos y enfrentarse a los problemas de la pareja. México: Paidós.

Bernal, A. (2001). La cifra de hombres maltratados es similar a la de mujeres. Extraído el 9 de diciembre de 2008 de www.diariodecanarias.com/maltratados007-eloy.htm.

Callirgos, J.C. (2003). Sobre héroes y batallas. Los caminos de la identidad masculina. En C. Lomas (Comp.). ¿Todos los hombres son iguales? Identidades masculinas y cambios sociales. (pp. 55-82). Barcelona: Paidós.

Connell, R. (2003). La organización social de la masculinidad. En Lomas, C. (Comp.) ¿Todos los hombres son iguales? Identidades masculinas y cambios sociales (pp. 11-31). Barcelona: Paidós.

Constenla, T. (2007, 29 de abril). Las chicas también pegan. Periódico El País. México, p. 27.

CORIAC (Colectivo de Hombres por Relaciones Igualitarias, AC). (1995). Inventario de conductas violentas. Minicarpeta sobre el trabajo con violencia masculina. México.

Corral, S. \& Calvete, E. (2006). Evaluación de la violencia en las relaciones de pareja mediante las "escalas de tácticas para conflictos": estructura factorial y diferencias de género en jóvenes. Psicología Conductual, 14 (2), 215-233.

Corsi, J. (1997). Una mirada abarcativa sobre el problema de la violencia familiar. En Corsi, J. (Comp.). Violencia familiar (pp. 45-63). Buenos Aires: Paidós.

Corsi, J. (1998). Abuso y victimización de la mujer en el contexto conyugal. Violencia doméstica. México: Cidhal-Centro para Mujeres. 
Del Ángel, K. (2003). No sólo esposas: también hay maridos golpeados. Extraído el 21 de noviembre de 2008 de www.contenido.com. $\mathrm{mx} / 2003 / a b r / m a r i d o s \_g o l p e a d o s . h t m$

Fiebert, M. (2004). Estudios de otros países ponen de manifiesto niveles similares de violencia en ambos miembros de la pareja. Extraído el 9 de febrero de 2009 www.amedirh.com.mx/apartados/articulos//art230604/violencimuj.htm

Goodrich, T., Rampage, C., Ellman, B. \& Halstead, K. (1989). Terapia familiar feminista. Barcelona: Paidós.

Hanson, K. (2005). El abuso sufrido por los jóvenes en los noviazgos con violencia. Extraído el 4 de noviembre de 2007 de www.nnadv.,org/ Newsletter/Summer04Spa.pdf

Hoff, B. (1998). 25473 Washington men a year. Extraído el 7 de marzo de 2008 de www.vix. com/menmag/gjdv.htm

Hoff, B. (1999). Why women assault: review of Fiebert, M. \& González, D. College women who initiate assaults on their male partners and the reasons offered for such behavior. Extraído el 22 de enero de 2008 de www. batteredmen.com/fiebertg.

Hoff, B. \& Easterbrooks, R. (1999). Ultrasensitive men and abusive relationships. Extraído el 9 de octubre de 2007 de www.vix.com/menmag/batultra.htm

Instituto Nacional de las Mujeres. (2004). Encuesta de Inmujeres DF. La violencia en el noviazgo se acrecienta en el matrimonio. Extraído el 13 de noviembre de 2005 de www.jornada.unam.mx/2004/oct04/041004/información/74_noviazgo.

Llerena, T. (2004). Abusos: cuando ellas pegan. Extraído el 17 de marzo de 2006 de www. geocities.com/adm_peru/medios07.htm

Lomas, C. (2003). Masculino, femenino y plural. En Lomas, C. (Comp.). ¿Todos los hombres son iguales? Identidades masculinas y cambios sociales. Barcelona: Paidós.

Menacho, C. (2004). ¿Sexo débil...? No, abusivas. Extraído el 17 de marzo de 2006 de www. geocities.com/adm_peru/medios05.htm

Monserrat, C. (2006, 11 de febrero). Una mujer mata a su marido de una puñalada en Huesca. Periódico El País. México, p. 27.

Montero, R. (2007, 20 de marzo). Nosotras. Periódico El País. España. Extraído el 21 de marzo de 2007 de www.elpais.com/articulo/ultima/ elpporopi/20070320elpepiult-2/Tes

Montesinos, R. (2002). Las rutas de la masculinidad. Ensayo sobre el cambio cultural y el mundo moderno. Barcelona: Gedisa.

Olamendi, P. (1997). La violencia contra la mujer en México. FEM, 171, 4-6.

Olszewski, P. (1999). Violent femmes. Extraído el 13 de febrero de 2001 de www.vix.com/menmag/violfemm.htm

Padres por la Igualdad Parental. (2000). [En línea] www.geocities.com/papahijo2000/vif.html [2001. abril 13].

Pérez, A. (2005). Violencia contra el hombre. Madrid: Libertad Digital.

Pérez, A. (2006). Violencia contra el hombre. Crónica negra. [En línea] www.findesemana.libertaddigital.com/articulo.php. [2007.enero 22]

Pérez, R. (2006, 11 de abril). La violencia, problema de salud pública. Periódico El País. p. 31. México.

Programa Amor es... sin violencia. (2005). [En línea] www.equidad.df.gob.mx/violencia/ amor_es.html [2006. febrero 2]

Ramírez, M. (2002). Hombres violentos. Un estudio antropológico de la violencia masculina. México: Plaza y Valdés. 
Rodríguez, K., Marín, M. \& Leone, M. (1993). El machismo en el imaginario social. Revista Latinoamericana de Psicología, 25 (2), 275-284.

Sacks, G. (2001). Domestic violence: a two-way street. Extraído el 16 de mayo de 2002 de www.batteredmen.com/batmaupin.htm

Saltijeral, T., Ramos, L. \& Caballero, A. (1998). Las mujeres que han sido víctimas de maltrato conyugal: tipos de violencia experimentada y algunos efectos en su salud mental. Salud Mental, 2, 10-18.

Seidler, V. (2003). Transformando las masculinidades. En Lomas, C. (Comp.). ¿Todos los hombres son iguales? Identidades masculinas y cambios sociales Barcelona: Paidós.

Straus, M. (2006). Bidireccional y simétrica: 111 estudios sobre la violencia en la pareja. Risk factors for physical violence between dating partners. Extraído el 16 de mayo de 2008 de http://www.psicologia-online.com/colaboradores/paola/violencia/index2.shtml

Sussman, M. \& Steinmetz, S. (1998). Handbook of marriage and the family. New York: Plenumm Press.

Trujano, P. (2008). Varones maltratados por sus mujeres: la otra cara de la violencia doméstica. En Vargas, M.I. (Comp.). Violencia Doméstica: ¿víctimas o cómplices? México: Porrúa.

Trujano, P., Martínez, K. \& Benítez, J. (2002). Violencia hacia el varón. Psiquis, 33(4), 5-19.
Trujano, P. \& Mata, E. (2002). Relaciones violentas en el noviazgo: un estudio exploratorio. Revista Psicología Conductual, 10 (2), 389-408.

Trujano, P. \& Mendoza, S. (2003). Violencia doméstica: estudio exploratorio acerca de su percepción y aceptación. Revista Iztapalapa, 54, 231-244.

Trujano, P., Nava, C., Tejeda, E. \& Gutiérrez, S. (2006). Estudio confirmatorio acerca de la frecuencia y percepción de la violencia doméstica: el VIDOFyP como instrumento de evaluación y algunas reflexiones psicosociales. Intervención Psicosocial, 15 (1), 95-110.

Vílchez, C. (2007). Violencia familiar o cuando los hombres son los únicos culpables: el efecto negativo de las leyes antifamiliares. Extraído el 2 de diciembre de 2007 de www.ordelise. org/index.php?option=com_content\&task=vi ew\&id=34\&/temid $=41$

Walker, E. (1984). The battered woman syndrome. New York: Springer.

Williamson, T. (1995). Hombres maltratados y violencia doméstica. Extraído el 29 de octubre de 1998 de www.redalyc.uaemex.mx/ redalyc/pdf/195/19500608.pdf

Zubizarreta, I., Sarasúa, B., Echeburúa, E., de Corral, P., Sauca, D. \& Emparanza, I. (1994). Consecuencias psicológicas del maltrato doméstico. En Echeburúa, E. (Comp.). Personalidades violentas. Madrid: Pirámide. 